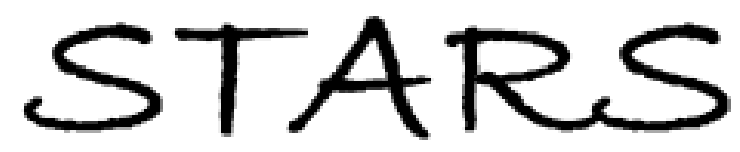

University of Central Florida

STARS

$1-1-2012$

\title{
Liquid crystal micro-lenticular array assembled by a fringing field
}

Miao Xu

Hongwen Ren

Changwoon Nah

Seung Hee Lee

Yifan Liu

University of Central Florida

Find similar works at: https://stars.library.ucf.edu/facultybib2010 University of Central Florida Libraries http://library.ucf.edu

This Article is brought to you for free and open access by the Faculty Bibliography at STARS. It has been accepted for inclusion in Faculty Bibliography 2010 s by an authorized administrator of STARS. For more information, please contactSTARS@ucf.edu.

\section{Recommended Citation}

Xu, Miao; Ren, Hongwen; Nah, Changwoon; Lee, Seung Hee; and Liu, Yifan, "Liquid crystal micro-lenticular array assembled by a fringing field" (2012). Faculty Bibliography 2010s. 3513.

https://stars.library.ucf.edu/facultybib2010/3513

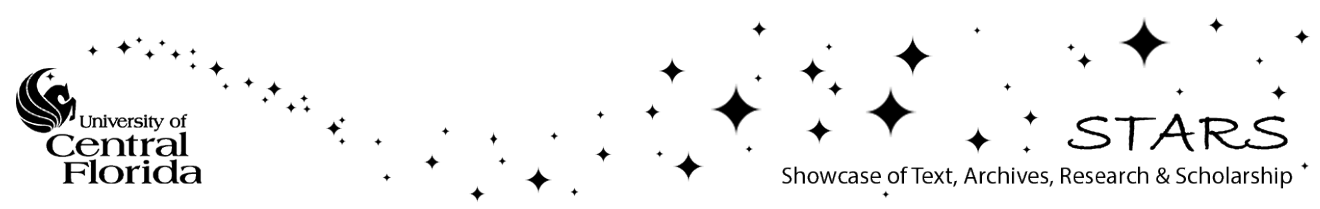




\section{Liquid crystal micro-lenticular array assembled by a fringing field}

Cite as: J. Appl. Phys. 111, 063104 (2012); https://doi.org/10.1063/1.3694738

Submitted: 30 November 2011 . Accepted: 18 February 2012 . Published Online: 21 March 2012

Miao Xu, Hongwen Ren, Changwoon Nah, Seung Hee Lee, and Yifan Liu

View Online

\section{ARTICLES YOU MAY BE INTERESTED IN}

Tunable-focus flat liquid crystal spherical lens

Applied Physics Letters 84, 4789 (2004); https://doi.org/10.1063/1.1760226

Tunable Fresnel lens using nanoscale polymer-dispersed liquid crystals

Applied Physics Letters 83, 1515 (2003); https://doi.org/10.1063/1.1604943

Field controlled light scattering from nematic microdroplets

Applied Physics Letters 48, 269 (1986); https://doi.org/10.1063/1.96577

\section{Applied Physics Reviews} Now accepting original research

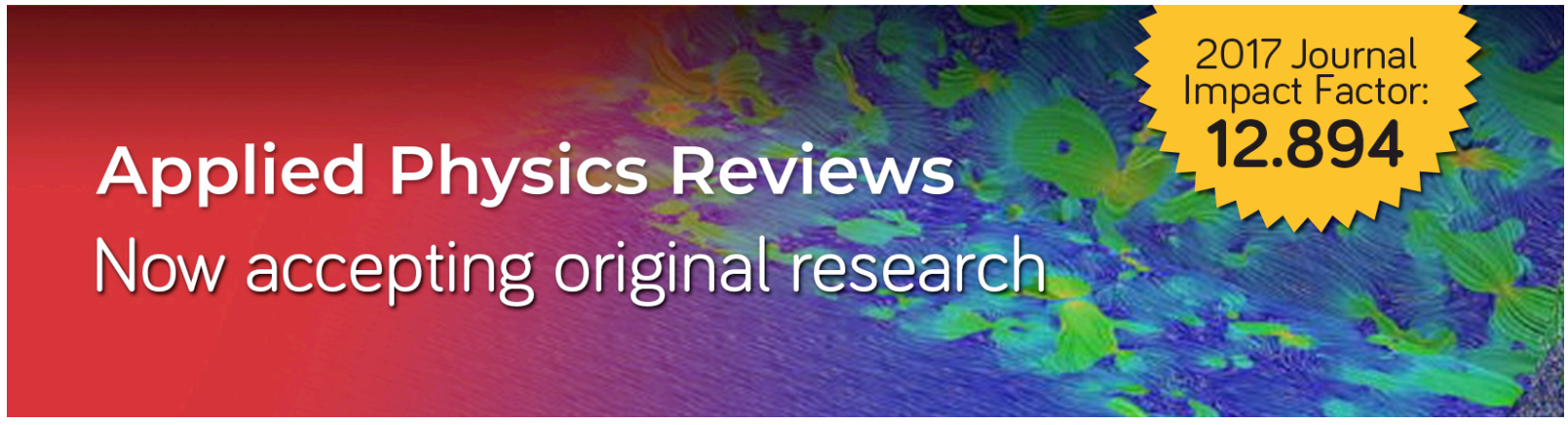




\title{
Liquid crystal micro-lenticular array assembled by a fringing field
}

\author{
Miao Xu, ${ }^{1}$ Hongwen Ren, ${ }^{1, a)}$ Changwoon Nah, ${ }^{1}$ Seung Hee Lee, ${ }^{1}$ and Yifan Liu ${ }^{2}$ \\ ${ }^{1}$ Department of Polymer-Nano Science and Technology, Chonbuk National University, Jeonju, \\ Chonbuk 561-756, Republic of Korea \\ ${ }^{2}$ College of Optics and Photonics, University of Central Florida, Orlando, Florida 32816, USA
}

(Received 30 November 2011; accepted 18 February 2012; published online 21 March 2012)

\begin{abstract}
Based on dielectrophoretic effect, the phase separation morphology of liquid crystal (LC) in a liquid monomer can be manipulated by a fringing field. Applying the fringing field generated from interdigitated electrode upon the LC/monomer mixture, the randomly dispersed LC droplets can be assembled to grating-like stripes. When the field is removed, the LC stripes break into tiny droplets again. This process is reversible and stable. Our results show that the surface profile of each LC stripe exhibits a lenticular shape. The response time of the LC morphology converting from droplets to stripes and from stripes to droplets is $\sim 0.96 \mathrm{~s}$. Potential applications of this controllable morphology can be found in optical communications, beam steering, imaging, and displays. (C) 2012 American Institute of Physics. [http://dx.doi.org/10.1063/1.3694738]
\end{abstract}

\section{INTRODUCTION}

Phase separation in a mixture of liquid crystal (LC) and prepolymer has been studied extensively. Thermally-induced polymerization and photo-induced polymerization are the two common ways for phase separation. ${ }^{1,2}$ As the phase separation completes, a LC-polymer composite film is formed. By choosing suitable materials as well as controlling the experimental conditions, various LC-polymer composite films can be obtained. ${ }^{3-10}$ These films have been widely used in switchable windows, ${ }^{3,4}$ variable optical attenuators, ${ }^{5}$ phase modulators, ${ }^{6}$ beam steering, ${ }^{7}$ scattering polarizer, ${ }^{8}$ and adaptive lenses. ${ }^{9,10}$ Because polymer in these LC-composite films has been solidified, their phase separation morphology could not be changed. To develop a new concept of electrooptical device, it is highly desirable that the phase separation morphology can be reassembled and controlled dynamically.

To control the phase separation morphology of LC and liquid monomer, dielectrophoresis is another viable approach. ${ }^{11-14}$ When the mixture of LC and liquid monomer is subject to a nonuniform electric field, LC molecules (or droplets) are pulled toward the region where the gradient of electric field is the highest, while the liquid monomers are pushed to the surrounded area. Based on this effect, West's group demonstrated monomer walls using a patterned electrode in a LC cell. ${ }^{14}$ Because the walls are used to maintain the cell gap, the monomers have to be polymerized using UV light. In another research, ${ }^{15}$ anisotropic phase separation between LC and liquid monomer by a fringing field has been investigated. Although in situ observation shows a significant impact of fringing field on LC morphology, little information is known about the dynamic response, the surface profile of the formed LC morphology, and the stability of the device.

In this work, we use a fringing field to control the phase separation of LC morphology. In the voltage-off state, LC is dispersed in a liquid monomer in the form of tiny droplets.

${ }^{a)}$ Electronic mail: hongwen@jbnu.ac.kr.
In a voltage-on state, these droplets are assembled to a stripe array by the fringing field. By removing the voltage, the stripe array breaks into droplets again. Our results show that the surface profile of each LC stripe exhibits a lenticular shape. LC morphology changing from droplets to lenticular array and from lenticular array to droplets gives a reasonable response speed. This type of device with controllable LC morphology from droplets to lenticular array has potential applications in waveguide, beam steering, imaging processing, and displays.

\section{BASIC THEORY AND SIMULATION}

It is well known that when a dielectric material is impacted by a non-uniform electric field, it bears a dielectric force. The dielectric force is expressed as ${ }^{1,12}$

$$
F=\frac{1}{2} \varepsilon_{0}\left(\varepsilon_{1}-\varepsilon_{m}\right) \nabla E^{2},
$$

where $\varepsilon_{0}$ represents the permittivity of free space, $\varepsilon_{1}$ and $\varepsilon_{m}$ represent the relative dielectric constants of the material and surrounded medium, respectively, and $\nabla E$ is the gradient of the electric field. If $\varepsilon_{1}>\varepsilon_{m}$, then the material will be attracted to the region with high $\nabla E$, while the surrounded medium is expelled to the place with low gradient of electric field. Such a dielectrophoretic effect has been widely used to manipulate dielectric particles, separate biological cells, move liquid droplets, and reconfigure the shape of a liquid surface.

From Eq. (1), the gradient of electric field plays an important role in the dielectric force. To generate a periodically nonuniform electrode field, interdigitated stripe electrode is preferred. The structure of our empty cell is shown in Fig. 1. The cell consists of top glass plate, indium tin oxide (ITO) electrode, and bottom glass plate. In Fig. 1, ITO exists only on bottom substrate. The ITO electrode is coated on the bottom glass surface and etched with a comb pattern. A coordinate is also given as well. The $x$-axis is perpendicular to the ITO stripe and $\mathrm{z}$-axis is along the cell gap direction. When a voltage is applied across the ITO stripes, fringing 


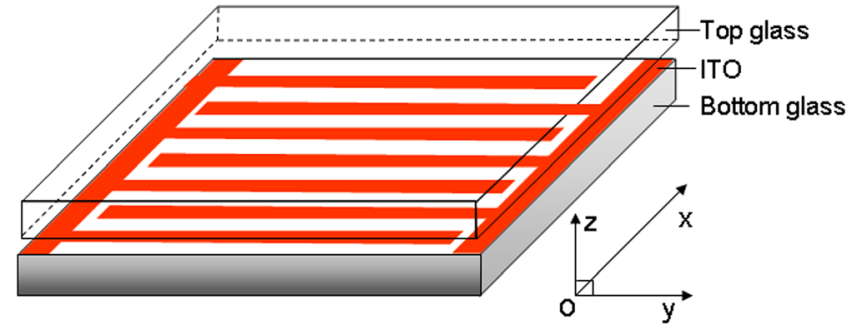

FIG. 1. (Color online) The structure of our empty cell with interdigited electrode strips. $y$-axis is along ITO strip direction and $z$-axis is perpendicular to the substrate surface.

fields are generated. The electric field distribution in the cell is simulated based on the cell structure as Fig. 1 shows. In our simulation, the width of ITO stripe, the ITO gap, and the cell gap are $5 \mu \mathrm{m}, 15 \mu \mathrm{m}$, and $20 \mu \mathrm{m}$, respectively. When a voltage is applied across the stripes, the electric field can be resolved into two components: along $x$-direction $\left(E_{x}\right)$ and along $z$-direction $\left(E_{z}\right)$. There is no electric field along $y$-direction. The electric field distribution along $x$-axis is shown in Fig. 2(a). The applied voltage is $\mathrm{V}=60 \mathrm{~V}_{\mathrm{rms}}$. Along $x$-direction, the electric field is the strongest near the edges of each ITO stripe. Such a result implies the dielectric force would point toward this region. The electric field distribution along $z$-axis is shown in Fig. 2(b). Above each ITO stripe but close to the electrode surface, the electric field is stronger than that in the surrounding area. The electric field distribution of another cell with $10-\mu \mathrm{m}$-width ITO stripe, $10-\mu \mathrm{m}$ ITO gap, and $20-\mu \mathrm{m}$ cell gap is also simulated. The result of electric field distribution is very similar to that shown in Fig. 2. According to Eq. (1), if two dielectric liquids with different dielectric constants are subject to the same fringing field, the liquid with larger dielectric constant will be attracted toward the surface of the ITO stripes.

\section{EXPERIMENTS}

To investigate the impact of fringing field on the LC morphology change, two empty cells (1 and 2) were prepared. The dimension of each cell is $1 \mathrm{~cm} \times 1.5 \mathrm{~cm}$. The ITO stripe width, ITO gap, and cell gap are $5 \mu \mathrm{m}, 15 \mu \mathrm{m}$, and $20 \mu \mathrm{m}$, respectively, for cell-1. As for cell-2, its ITO stripe width, ITO gap and cell gap are $10 \mu \mathrm{m}, 10 \mu \mathrm{m}$, and $20 \mu \mathrm{m}$, respectively. LC (SLC132-010, $n_{e}=1.78, n=0.265, \varepsilon_{/ /} \sim 26.1$,
$\Delta \varepsilon \sim 20$, Chengzhi Yonghua Display Material, China) and liquid monomer NOA65 (Norland adhesive $\varepsilon_{p} \sim 5, n_{p}=1.52$ ) were chosen as the experimental materials. The two materials are partially soluble. Two mixtures were prepared: mixture-1 contained 48 wt.\% SLC132-010 and 52 wt.\% NOA65 while mixture-2 contained 65 wt.\% SLC132-010 and 35 wt.\% NOA65. Mixture-1 was filled in cell-1 and mixture-2 was filled in cell-2.

\section{RESULTS AND DISCUSSION}

We first investigated the phase separation morphology of cell-1 using a polarizing optical microscope (POM). The cell was placed between crossed polarizers on the POM stage. Initially the phase separation happened spontaneously at room temperature. Only a small amount of LC was separated from the monomer in the form of droplets. These LC droplets were randomly dispersed in the monomer as Fig. 3(a) shows. When a voltage of $\mathrm{V}=70 \mathrm{~V}_{\mathrm{rms}}$ was applied to the electrodes, the LC droplets experienced a dielectric force. Since the dielectric constant $\left(\varepsilon_{/ / /} \sim 26\right)$ of LC is much larger than that of monomer $\left(\varepsilon_{p} \sim 5\right)$, these LC droplets were attracted to the ITO stripes and became elongated on the edges of each ITO stripe, as shown in Fig. 3(b). Each LC line consists of several segments because the amount of LC is insufficient for them to be connected together. In comparison to Fig. 3(a), the amount of LC in Fig. 3(b) is larger. This is perhaps because some LC is separated from the monomer by the dielectric force. When the voltage is decreased to $60 \mathrm{~V}_{\text {rms }}$, the LC morphology does not change evidently except that each LC segment shrinks a little bit, as depicted in Fig. 3(c). Such a result implies the voltage of $60 \mathrm{~V}_{\mathrm{rms}}$ is high enough for forming the striped LC morphology. As the voltage is reduced to $50 \mathrm{~V}_{\mathrm{rms}}$, each LC segment shrinks significantly (Fig. 3(d)). The contraction of LC segments results from the balance between LC surface tension and dielectric force. Continuously decreasing the voltage to $20 \mathrm{~V}_{\text {rms }}$, each LC segment shrinks to "8" shape (Fig. 3(e)). When the voltage is removed, each LC segment returns to the original droplet and a well-regulated droplet array is formed (Fig. 3(f)). If the voltage is again gradually increased from zero to $70 \mathrm{~V}_{\text {rms }}$, the LC morphology would repeat following the order from Figs. 3(e) $\rightarrow 3$ (d) $\rightarrow 3$ (c) $\rightarrow$ 3 (b). In each case, the formed LC morphology is very stable if the applied voltage is fixed.
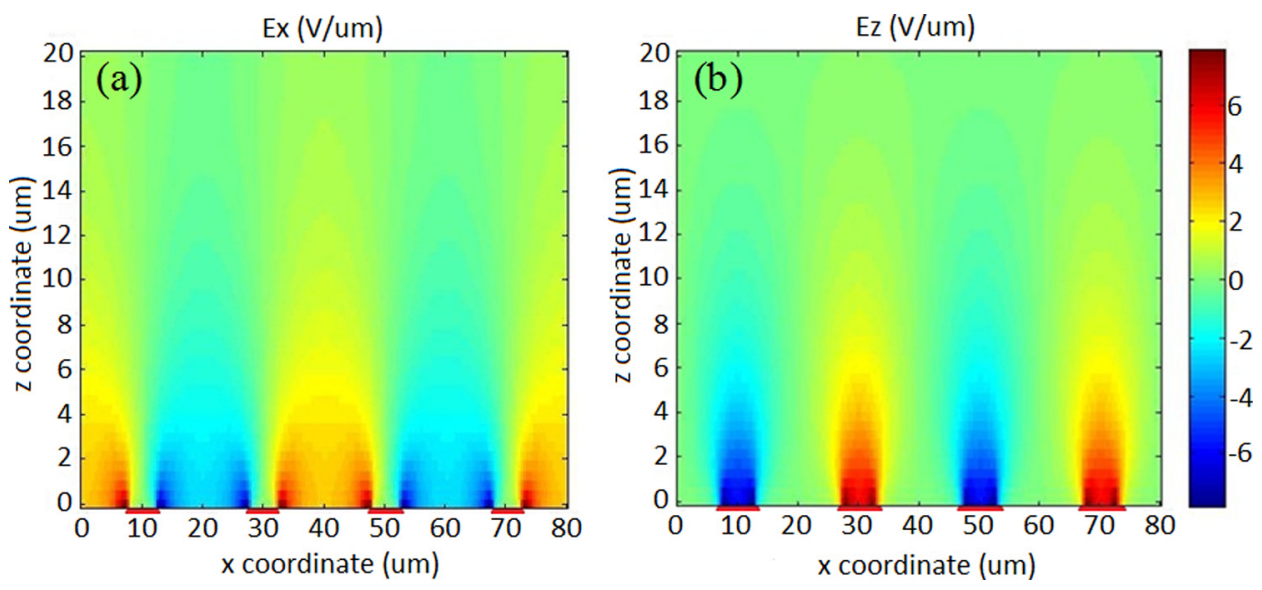

FIG. 2. (Color online) The simulated electric-field distribution. (a) electric field $\left(E_{x}\right)$ in $x$-direction and (b) electric field $\left(E_{z}\right)$ in $z$-direction. The width of ITO strip is $5 \mu \mathrm{m}$, the gap of the adjacent strips is $15 \mu \mathrm{m}$, and the cell gap is $20 \mu \mathrm{m}$. The cell gap and the ITO width are not drawn to scale. 

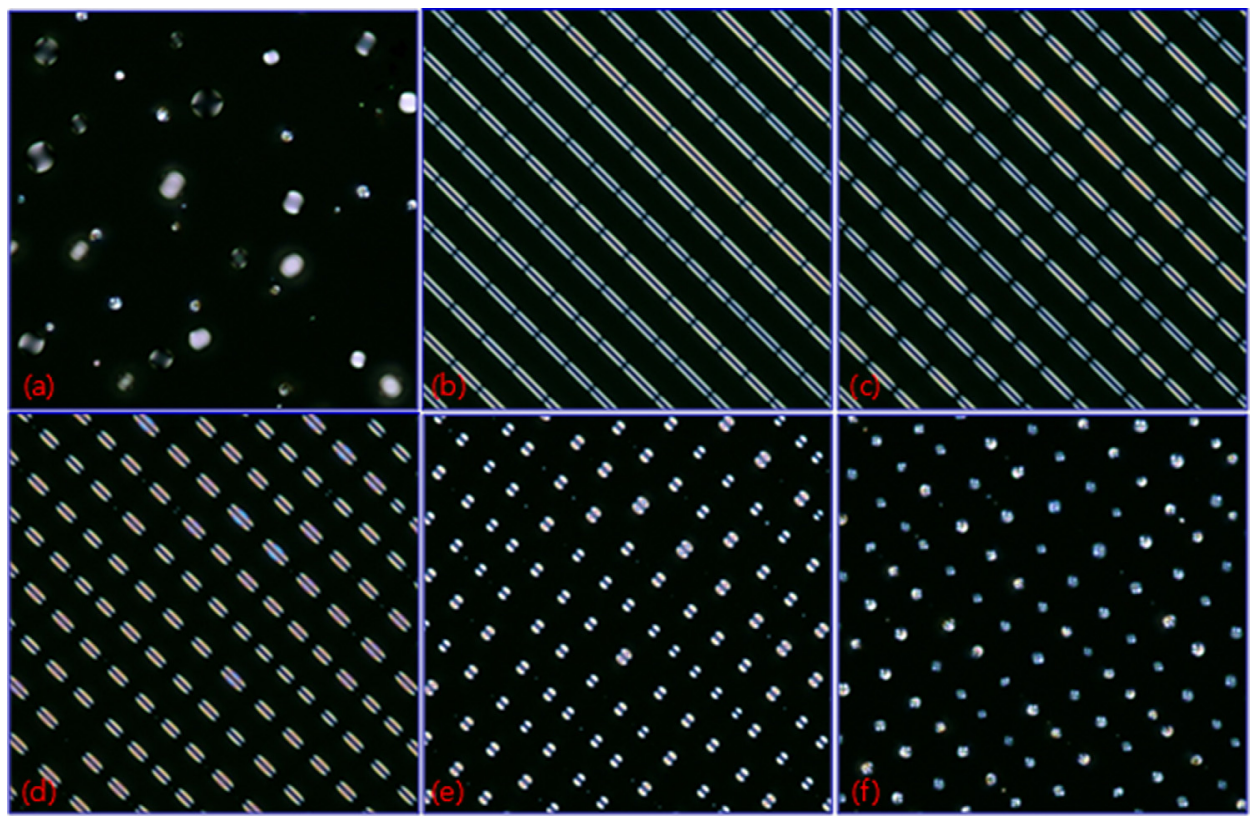

FIG. 3. (Color online) Top view of phase separated morphologies of LC in cell-1 driven with different voltages. (a) $\mathrm{V}=0$, (b) $\mathrm{V}=70 \mathrm{~V}_{\mathrm{rms}}$, (c) $\mathrm{V}=60 \mathrm{~V}_{\mathrm{rms}}$, (d) $\mathrm{V}=50 \mathrm{~V}_{\text {rms }}$, (e) $\mathrm{V}=20 \mathrm{~V}_{\text {rms }}$, and (f) $\mathrm{V}=0$.

Why different LC morphologies occur at different voltages can be explained in Fig. 4. According to the simulation results shown in Fig. 2, when a voltage is applied to the ITO stripes, the region near the stripe edges provides the largest gradient electric field $\left(E_{z}\right.$ and $\left.E_{x}\right)$. LC is preferred to fill this region, as depicted in Fig. 4(a) (left). In the central area of ITO stripe, the gradient of electric field is relatively weak. This region is occupied by monomer if the LC amount separated from the monomer is not enough. Therefore, two LC lines are formed on the edges of an ITO stripe as the phase separation is complete (Fig. 4(a) (right)). Such explanation is supported by the observed result shown in Fig. 3(c). If the amount of LC separated from the monomer is large enough, then $\mathrm{LC}$ in the two regions can grow up quickly and combine together due to the surface tension, as shown in Fig. 4(b) (left). As the phase separation is over, it is possible to form a LC lenticular stripe, as depicted in Fig. 4(b) (right).

Since the LC morphology can be converted from droplets (stripe) to stripe (droplets), it is convenient for us to estimate the morphology transition response through light diffraction. Here, we use a He-Ne laser beam $(\lambda=633 \mathrm{~nm})$ to probe the morphology change. A $60 \mathrm{~V}_{\mathrm{rms}}$ (square-wave) is used to impact the LC droplets. Due to the grating-like structure (Fig. 3(c)), a photodiode detector can be used to detect the first-order diffraction beam. The measured light intensity by the detector is analyzed using a digital oscilloscope. Results are plotted in Fig. 5.

In Fig. 5, two cycles of driving with the same pulse widths are given. At $\mathrm{V}=0$, no grating effect from the randomly dispersed LC droplets is observed. Thus, the received light intensity is very weak. As the applied voltage increases to $60 \mathrm{Vs}$, the detected light intensity increases quickly and then gradually saturates. The saturated light intensity implies that the grating morphology has been formed. It takes $\sim 0.79$ $\mathrm{s}$ for the fringing field to complete the grating transition. After the impact of the pulse, it takes $\sim 0.17 \mathrm{~s}$ for the grating to collapse into LC droplets again. Two cycles give the same electro-optical curves. These results imply that the LC morphology can be well reassembled between droplets and stripes.

From Fig. 4, it would be attractive if LC droplets can be converted to a lenticular stripe at a lower operating voltage. To examine this possibility, it is worthwhile to study the morphology of cell-2 since it owns a wider ITO stripe and contains 65 wt.\% LC. Similar to cell-1, the morphology of cell-2 was observed using the POM between crossed polarizers. Figure 6 shows the LC morphologies of cell-2 with different voltages. At $\mathrm{V}=0$, the $\mathrm{LC}$ separated from liquid
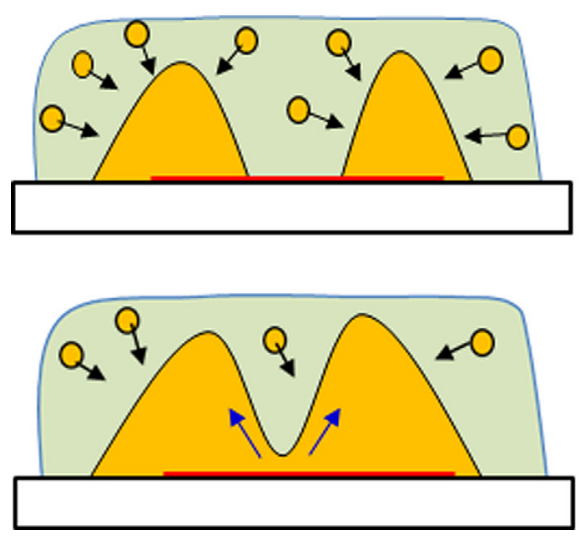

(a)

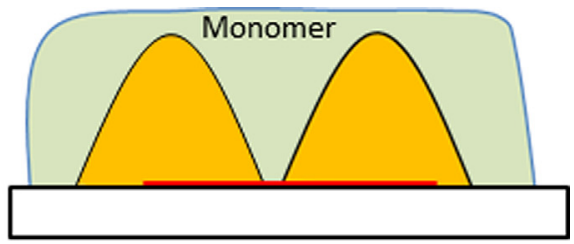

(b)

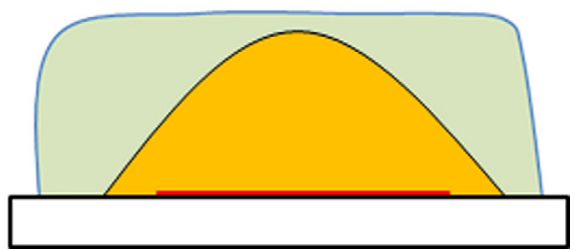

FIG. 4. (Color online) Phase separation morphology of LC with low (a) and high (b) LC concentrations in a liquid monomer. The left: during phase separation. The right: completed morphology. 


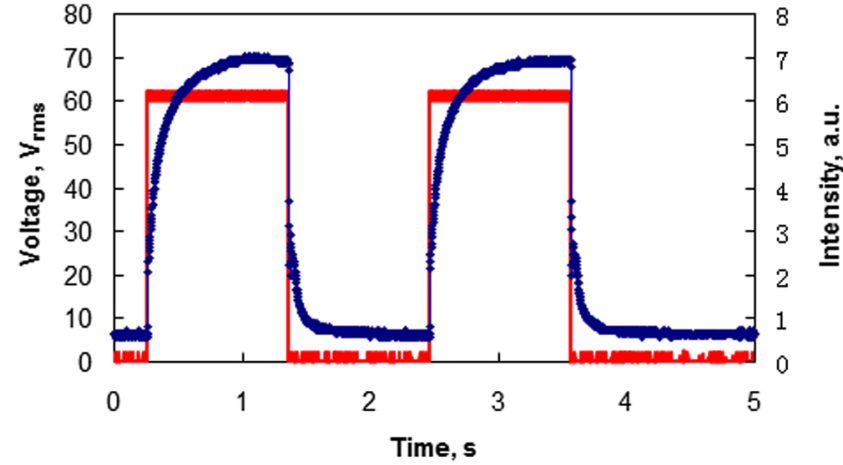

FIG. 5. (Color online) Dynamic response of the LC morphology assembled from droplets to strips and from strips to droplets. The amplitude and width of the voltage pulse is $60 \mathrm{~V}_{\mathrm{rms}}$ and $1 \mathrm{~s}$, respectively. The squared line voltage pulse, the curved line: light intensity.

monomer forms large droplets (Fig. 6(a)). When a voltage is applied to the ITO stripes and increased gradually, the LC droplets are stretched along ITO stripes. At $40 \mathrm{~V}_{\text {rms }}$, most of LC droplets are assembled to exhibit a partially grating-like structure (Fig. 6(b)). From the displayed color (green in the center and pink at the border), LC molecules are aligned well by the fringing field. As the voltage is increased to $50 \mathrm{~V}_{\mathrm{rms}}$, a grating-like morphology is achieved around the whole cell (Fig. 6(c)). At $60 \mathrm{~V}_{\mathrm{rms}}$, the LC morphology does not change too much (Fig. 6(d)). The uniform color implies that LC molecules exhibit uniform alignment by the fringing field. When the voltage is removed, the LC stripes collapse into LC droplets again. The dynamic response time of cell-2 is similar to that of cell-1.

To evaluate the shape of the LC stripes as shown in Fig. 6(d), the cell was fully cured using UV light with a bias voltage of $\mathrm{V}=60 \mathrm{~V}_{\mathrm{rms}}$. After $\mathrm{UV}$ exposure, the liquid

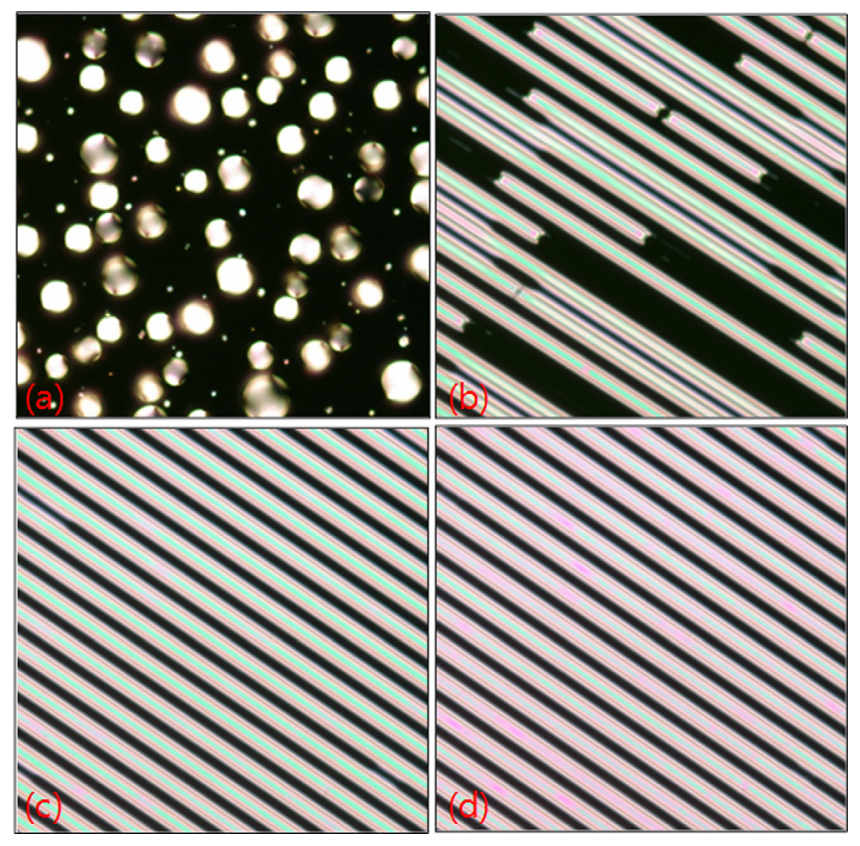

FIG. 6. (Color online) Phase separation morphology of 65\% LC impacted with different voltages. (a) $\mathrm{V}=0$, (b) $\mathrm{V}=40 \mathrm{~V}_{\text {rms }}$, (c) $\mathrm{V}=50 \mathrm{~V}_{\text {rms }}$, and (d) $\mathrm{V}=60 \mathrm{~V}_{\mathrm{rms}}$. A video of dynamic phase separation is also provided. The voltage was gradually increased from 0 to $70 \mathrm{~V}_{\mathrm{rms}}$ and switched off (enhanced online). [URL: http://dx.doi.org/10.1063/1.3694738.1] monomer was solidified which can record the surface profile of the LC stripes. The cell was opened and the LC was cleansed using ethanol solvent. When the cured polymer was dried, its surface was coated with an ultra-thin silver film. The surface profile of the polymer was detected using scanning electron microscope (SEM). Figure 7 shows the observed surface profile of the solidified polymer. The surface of the polymer exhibits concave-lenticular profile and the curved surface is rather smooth. Such a result proves that the formed LC stripes in Fig. 6 exhibit lenticular pattern.

From Fig. 7, the volume of the cavities occupies the whole space can be estimated if the shape of each cavity is considered to be spherical. Knowing the concentration of LC in its mixture (65 wt.\%), the thickness of the cell gap $(\sim 20$ $\mu \mathrm{m})$, and the aperture of the lenticular cavity $(\sim 15 \mu \mathrm{m})$, the cavity occupies the whole space is calculated to be $\sim 22 \%$. Such a result means $\sim 22 \%$ volume of LC has separated from the liquid monomer. Because the density of LC matches that of the monomer, $\sim 43 \%$ volume of $\mathrm{LC}$ remains in the liquid monomer. To force more LC to separate from the monomer, a common way is to increase the voltage in order to enhance the generated dielectric force. Another effective way is to decrease the cell gap, so that the generated dielectric force can highly affect the bulk of the cell during phase separation. Similarly, the volume of LC occupies the whole space of the cell (Fig. 3(b)) also can be estimated. At V=60 $\mathrm{V}_{\text {rms }}$, the separated LC only occupies $\sim 8 \%$ of the whole bulk. Such low volume ratio is mainly due to the large ITO gap $(15 \mu \mathrm{m})$ and low LC concentration. To get a suitable LC lenticular array, LC concentration in its mixture should be optimized. If the LC concentration is too low, then it is difficult to broaden the aperture of the formed LC stripes. On the contrary, if the LC concentration is too high, then the monomer could not form a concave lenticular around the LC stripes.

To avoid any polymerization, it is better to choose a suitable liquid to replace the UV curable monomer. To pickup a suitable liquid, the liquid should have the properties of low dielectric constant $(\varepsilon<5)$, low viscosity, optical isotropy, UV stable, and partial soluble with LC. Many liquid monomers (such as epoxy) are satisfied with these properties.

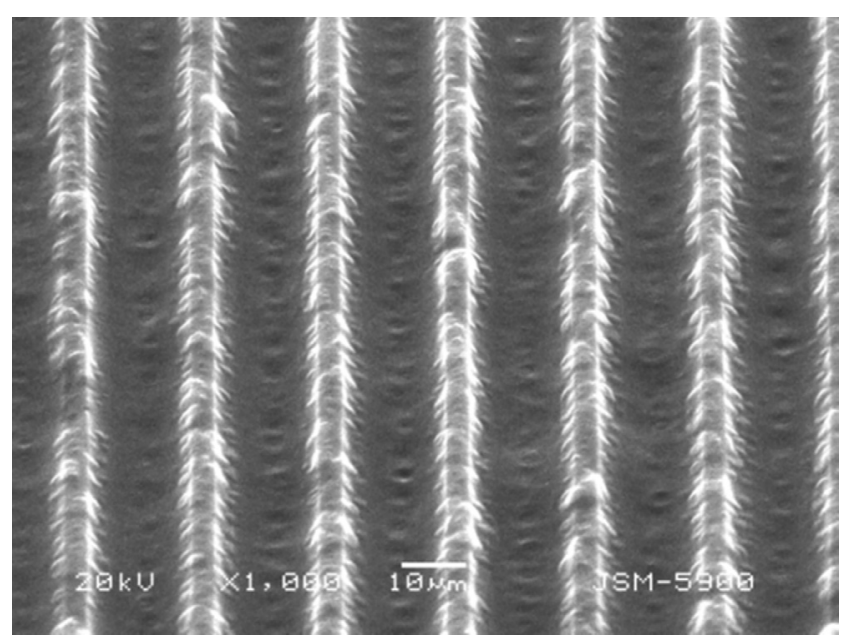

FIG. 7. The shape of the solid polymer surface taken using SEM. The concave-lenticular array is the region where the separated LC has occupied. 
From our experiment, nonuniform LC droplets can be converted to very uniform stripes. Once the LC stripes breakup without the support of fringing field, they can form relatively small and uniform droplets. These isolate droplets suspend in the liquid but close to the ITO stripes. The formed LC morphology is stable. Response speed of the morphology converting from droplets to stripes is mainly dependent on the generated dielectric force and the viscosity of the materials. A higher voltage usually generates a larger gradient of electric field. As a result, faster response can be achieved. To decrease the operating voltage while still keeping the same dielectric force, the gap of the adjacent ITO stripes should be decreased further.

The LC morphology switchable from droplets to lenticular stripes has potential applications in optical switch, beaming steering and imaging. For instance, one LC lenticular stripe can be considered as a waveguide because the average refractive index of LC $\left(n_{\text {eff }}\right)$ is larger than that of the liquid monomer and the orientation of LC is perpendicular to the LC stripe. Once the voltage is removed, the waveguide disappears and light will be strongly scattered by the LC droplets. As a beam steering, it is better to pattern the interdigitated electrode to be curved rather than straight, so that a curved LC channel can be assembled by the generated dielectric force. As a result, a beam can be steered by the curved channel. Because of the lenticular shape, the formed LC stripe also bears a lens character. Therefore, the formed LC micro-lenticular array can be used in imaging processing and three-dimensional displays.

\section{CONCLUSION}

We have demonstrated the feasibility of manipulating the morphology of LC and liquid monomer using a fringing field. Without an external voltage, LC droplets disperse in the monomer. When a fringing field is acted upon the LC droplets, a grating-like LC stripes are formed. By removing the voltage, the LC stripes collapse into droplets again due to the attraction of surface tension. Multi-cycle voltage driving shows that LC morphology can be reassembled very well. The transition time from droplets to stripes is $\sim 0.79 \mathrm{~s}$ and the collapsing time from stripes to droplets is $0.17 \mathrm{~s}$. SEM shows that each LC stripe exhibits a lenticular shape. Reassembled LC morphology from droplets to lenticular stripe makes the device attractive for optical switch, beam steering, imaging, and displays.

This work is supported by the National Research Foundation of Korea, the Basic Science Research Program (2010-0021680).

${ }^{1}$ P. S. Drzaic, Liquid Crystal Dispersions (World Scientific, Singapore, 1995).

${ }^{2}$ J. W. Doane, Liquid Crystals, Applications and Uses, edited by B. Bahadur (World Scientific, Singapore, 1990), Vol. 1, Ch. 14.

${ }^{3}$ J. L. West, Mo. Cryst. Liq. Cryst. 157, 427 (1988).

${ }^{4}$ D. K. Yang, L. C. Chien, and J. W. Doane, Appl. Phys. Lett. 60, 3102 (1992).

${ }^{5}$ K. Takizawa, K. Kodama, and K. Kashi, Appl. Opt. 37, 3181 (1998).

${ }^{6}$ S. Matsumoto, K. Hirabayashi, S. Sakata, and T. Hayashi, IEEE Photonics Technology Lett. 11, 442 (1999).

${ }^{7}$ T. J. Bunning, L. V. Natarajan, V. P. Tondiglia, and R. L. Sutherland, Annu. Rev. Mater. Sci. 30, 83 (2000).

${ }^{8}$ R. A. M. Hikmet and H. M. J. Boots, Phys. Rev. E 51, 5824 (1995).

${ }^{9}$ H. S. Ji, J. H. Kim, and S. Kumar, Opt. Lett. 28, 1147 (2003).

${ }^{10}$ H. Ren and S. T. Wu, Appl. Phys. Lett. 82, 22 (2003).

${ }^{11}$ P. Penfield and H. A. Haus, Electrodynamics of Moving Media (MIT, Cambridge, 1976).

${ }^{12}$ H. A. Pohl, Dielectrophoresis (Cambridge University Press, 1978).

${ }^{13}$ Y. Kim J. Francl, B. Taheri, and J. L. West, Appl. Phys. Lett. 72, 2253 (1998).

${ }^{14}$ H. Ren, S. T. Wu, and Y. H. Lin, Appl. Phys. Lett. 90, 191105 (2007).

${ }^{15}$ H. Ren, S. T. Wu, and Y. H. Lin, Phys. Rev. Lett. 100, 117801 (2008). 